\title{
Archivos

\section{Tratamiento endovascular exitoso del síndrome de cascanueces con stent autoexpandible}

\author{
Marco Antonio Alcocer-Gamba a,b,*, Jorge A. Martínez-Chávez ${ }^{\mathrm{c}}$, \\ Mónica Alcántara-Razo ${ }^{d}$, Guering Eid-Lidte, Leslie M. Lugo-Gavidia ${ }^{b}$, \\ Enrique García-Hernández ${ }^{a}$, Aquiles Montalvo-Ramos ${ }^{f}$, \\ Ivonne A. Torres-Quiroz ${ }^{\mathrm{g}}$ y Arturo Velázquez-Verduzco ${ }^{\text {b }}$
}

\footnotetext{
a Cardiología Intervencionista, Instituto de Corazón de Querétaro, Querétaro, Qro, México

b Facultad de Medicina, Universidad Autónoma de Querétaro, Querétaro, Qro, México

c Servicio de Urología, Hospital Médica Tec100 de Querétaro, Querétaro, Qro, México

d Servicio de Imagenología, Hospital Médica Tec100 de Querétaro, Querétaro, Qro, México

e Cardiología Intervencionista, Instituto Nacional de Cardiología Ignacio Chávez, México D.F., México

f Cardiología intervencionista, Hospital Médica Tec100 de Querétaro, Querétaro, Qro, México

g Investigación clínica, Instituto de Corazón de Querétaro, Querétaro, Qro, México
}

Recibido el 9 de diciembre de 2011; aceptado el 27 de septiembre de 2012

\author{
PALABRAS CLAVE \\ Síndrome de \\ cascanueces; \\ Vena renal izquierda; \\ Hematuria; \\ Tratamiento \\ endovascular; \\ Stent \\ autoexpandible; \\ México
}

\begin{abstract}
Resumen El síndrome de cascanueces (nutcracker's syndrome) se produce por compresión de la vena renal izquierda a su paso por la horquilla vascular, formada por la aorta y la arteria mesentérica superior, causando una compresión extrínseca que genera estenosis funcional de la misma. Esto produce congestión e hipertensión de la vena renal izquierda que se traduce en insuficiencia y várices de la vena gonadal izquierda, hematuria unilateral y dolor lumbar izquierdo, el diagnóstico pocas veces se realiza, por su baja frecuencia y por la falta de sospecha clínica. El tratamiento del síndrome de cascanueces incluye el autotransplante renal, trasposición de arteria mesentérica superior, revascularización y más recientemente, la colocación de stent en la vena renal. Presentamos el caso de un paciente que fue sometido a tratamiento endovascular exitoso con un stent autoexpandible en la vena renal izquierda, con criterios inmediatos de éxito por angiografía, reducción de la congestión venosa y por desaparición del gradiente cavo/renal.

(c) 2011 Instituto Nacional de Cardiología Ignacio Chávez. Publicado por Masson Doyma México S.A. Todos los derechos reservados.
\end{abstract}

\footnotetext{
* Autor para correspondencia. Instituto de Corazón de Querétaro, Prol. Priv. Ignacio Zaragoza 16B 2 ${ }^{\circ}$ piso, Colonis Centro, C.P. 76000, Querétaro, Qro, México. Teléfono: +442 2162745, 2162746; fax: +442 2164922.

Correo electrónico: marco.alcocerg@gmail.com (M.A. Alcocer-Gamba).
} 


\section{KEYWORDS}

Nutcracker's

syndrome;

Left renal vein;

Hematuria;

Endovascular

treatment;

Self-expanding stent;

Mexico
Successful endovascular treatment of nutcracker's syndrome with self-expanding stent

Abstract Nutcracker's syndrome is caused by compression of the left renal vein between aorta and superior mesenteric artery, causing extrinsic compression generated functional stenosis. This causes congestion and hypertension of the left renal vein resulting in insufficiency and left gonadal vein varicose, unilateral hematuria and left flank pain, diagnosis is rarely identified by their low frequency and difficulty of suspecting, treatment of nutcracker's syndrome include renal autotransplantation, transposition of superior mesenteric artery revascularization and recently stenting in renal vein, we present the case of a patient, who showed this pathology by diagnostic support by image studies, was performed successfully implant a self-expanding stent with immediate success criteria by angiography, collateral reduction and by disappearance of cava/renal gradient.

(C) 2011 Instituto Nacional de Cardiología Ignacio Chávez. Published by Masson Doyma México S.A. All rights reserved.

\section{Introducción}

El síndrome del cascanueces es una compresión extrínseca de la vena renal izquierda producida como consecuencia de su atrapamiento entre la aorta y la arteria mesentérica superior, a nivel del compás aorto-mesentérico, esto genera hipertensión de la vena renal izquierda que se traduce en insuficiencia y várices de la vena gonadal izquierda y hematuria unilateral; fue descrito de manera inicial por De Screpper en 1972, como "síndrome de atrapamiento de la vena renal izquierda"'1. El fenómeno del cascanueces, se refiere a la compresión anatómica de la vena renal izquierda entre la arteria mesentérica superior y la aorta abdominal, dando como resultado la limitación en la salida de dicha vena. El fenómeno del cascanueces se debe de diferenciar del síndrome del cascanueces, ya que éste último describe los síntomas asociados y demostrados por los cambios anatómicos del fenómeno de cascanueces ${ }^{2}$. Constituye una causa poco común de hematuria procedente del sistema colector izquierdo secundaria a dicha compresión, que ocasiona incremento de la presión del sistema venoso renal izquierdo, con el posterior desarrollo de varicosidades a nivel de la pelvis renal y uréter, que comunican con la vía excretora, dando lugar a episodios de hematuria ${ }^{1}$. Clínicamente es posible que permanezca silente o se manifieste como episodios de hematuria macro o microscópica, la cual puede ir acompañado de dolor en fosa renal izquierda y/o dolor abdominal ${ }^{3,4}$. Es posible que existan también episodios de albuminuria recurrente en jóvenes, que se incrementa con el ortostatismo, así como con el síndrome de fatiga crónica, que debe sospecharse en aquellos niños con desórdenes psicosomáticos, hipotensión ortostática, taquicardia postural $\mathrm{u}$ otros síntomas de disfunción autonómica ${ }^{5,6}$. Puede cursar con varicocele izquierdo y várices lumbares, debido a que el incremento de presión a nivel de la vena renal izquierda se transmite de modo retrógrado por la vena gonadal del mismo lado, esto produce un incremento de presión a nivel del plexo pampiniforme, tributario de la vena gonadal, con el desarrollo de varicocele izquierdo en diferentes grados, esta obstrucción al flujo gonadal también se relaciona con síntomas congestivos pélvicos. Es una patología de difícil diagnóstico, existen menos de 200 casos publicados en la literatura médica, su diagnóstico es infrecuente, tanto por su baja incidencia, como por la dificultad de sospecharlo ante hallazgos tan frecuentes como dolor, hematuria, varicocele o síntomas urinarios bajos o pelvianos; inicialmente debe realizarse un estudio detallado de hematuria para descartar otras causas más frecuentes. La flebografía se le considera una técnica de elección, puesto que nos permite llevar a cabo cálculos de gradientes de presión entre la porción distal de la vena renal y la vena cava inferior (normal 0-1 mmHg), no obstante, el cálculo de este gradiente no es imprescindible para el diagnóstico, debido a que pueden existir variaciones del mismo en función del grado de circulación colateral existente ${ }^{7}$. El diagnóstico se identifica en el ultrasonido (US) Doppler, tomografía computada (TC) y/o resonancia magnética (RM). La TC aporta información anatómica sobre la relación de la vena renal izquierda con la aorta y la arteria mesentérica superior, con lo que se establece el diagnóstico de síndrome del cascanueces $^{7}$. El tratamiento del síndrome del cascanueces depende de la severidad de los síntomas, aquellos pacientes con síntomas leves o tolerables pueden ser tratados en forma conservadora. Las opciones terapéuticas disponibles en la actualidad pueden subclasificarse en 3 grupos: 1) vigilancia, tratamiento propuesto principalmente en hematuria moderada $^{8}$, ya que la resolución espontánea suele ocurrir en estos $\left.\operatorname{casos}^{9}, 2\right)$ procedimientos quirúrgicos abiertos: nefropexia medial, derivación de la vena renal, trasposición de la vena renal izquierda y autotrasplante de riñón izquierdo (estos procedimientos más agresivos se pueden requerir si la hematuria es severa y causa anemia o existe dolor abdominal o en el flanco izquierdo incapacitante) ${ }^{8}$, y 3) stents intravasculares: esta es una alternativa mínimamente invasiva, con resultados favorables y menor morbilidad, aunque su eficacia y seguridad debe ser comprobada a largo plazo. Hay diversos reportes como el de Shanwen Chen publicado en el 2011, en 61 pacientes con implante de stent autoexpandible, el cual demostró que se trata de un procedimiento seguro que ofrece resultados beneficiosos para los pacientes a corto plazo y sugieren la terapia intravascular como la mejor opción terapéutica ${ }^{8,10}$, las complicaciones a considerar en este procedimiento son principalmente dadas por la migración del stent ${ }^{10}$. 


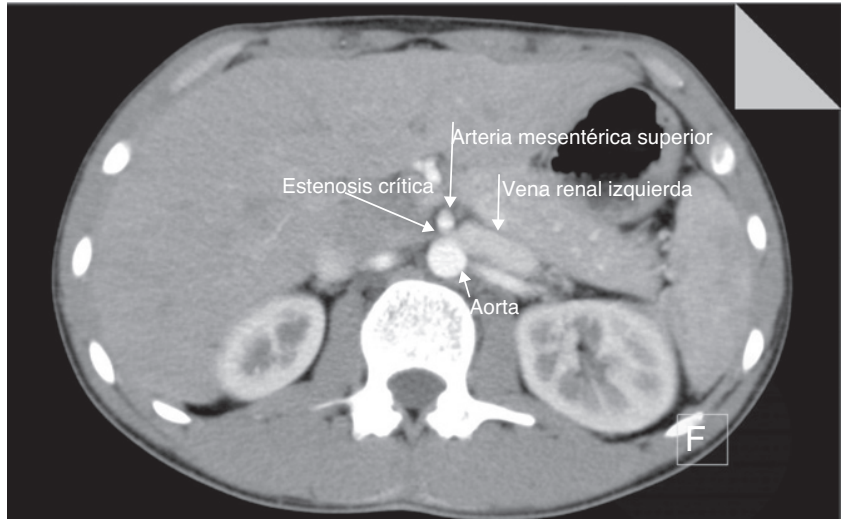

Figura 1 Corte axial de tomografía computada con contraste endovenoso en fase arterial, donde se demuestra la compresión de la vena renal izquierda entre la aorta y la emergencia de la arteria mesentérica superior con dilatación retrógrada.

\section{Presentación del caso}

Presentamos el caso de un joven varón de 19 años de edad, sin antecedentes clínicos, quirúrgicos, ni urológicos de relevancia, que inició en junio del 2011, con hematuria total silenciosa, constante, sin fiebre, ni otros datos. A la exploración física endomórfico, presión arterial de $100 / 70 \mathrm{mmHg}$, ruidos cardiacos rítmicos y sin alteraciones, campos pulmonares limpios, abdomen sin alteraciones, varicocele izquierdo grado III, en decúbito, con disminución del tamaño y consistencia del testículo de ese lado. Los estudios de laboratorio como biometría hemática, azoados, ácido úrico, perfil de lípidos, pruebas de función hepática y electrolitos séricos se reportaron en límites normales, el examen general de orina con proteinuria $>200 \mathrm{mg} / \mathrm{dL}$, hematuria (> $3+)$, US renal sin lesión demostrable, flujo renal aumentado, vejiga sin residuo. La TC con riñón izquierdo de $12.1 \mathrm{x}$ $5.4 \times 5 \mathrm{~cm}$, arteria renal única con bifurcación a $2.5 \mathrm{~cm}$ del origen en la aorta y diámetro máximo de $6 \mathrm{~mm}$, vena renal izquierda única, ectásica en la porción intrarrenal, trayecto preaórtico con adelgazamiento significativo a nivel de la emergencia de la arteria mesentérica superior, con un diámetro anteroposterior de $2 \mathrm{~mm}$, la desembocadura de la vena testicular en la vena renal izquierda está ectásica, zigzagueante, con formación de colaterales que van hasta la bolsa escrotal izquierda (fig. 1). El análisis espectral del Doppler en la vena renal izquierda a nivel del hilio, no mostró variación de flujo con los movimientos respiratorios con inversión de espectro y velocidad de $21.3 \mathrm{~cm} / \mathrm{seg}$, las venas arcuatas segmentarias mostraron el mismo patrón, a nivel del hilio se identificó compresión o atrapamiento de dicha vena en el espacio aorto-mesentérico, en la región testicular se identificó ectasia del plexo pampiniforme con eje transverso de $4.1 \mathrm{~mm}$, en la cual se identificó importante estasis sanguínea siguiendo colaterales a nivel de la corredera parietocólica y flujo anterógrado con variante de respiración (fig. 2), se concluyó el diagnóstico de síndrome de hipertensión de venal renal izquierda con síndrome de vena gonadal, varicocele grado IV y presencia de síndrome de atrapamiento de la vena renal izquierda.

Se concluyó el diagnóstico de síndrome del cascanueces, se solicitó realizar estudio angiográfico por vía femoral

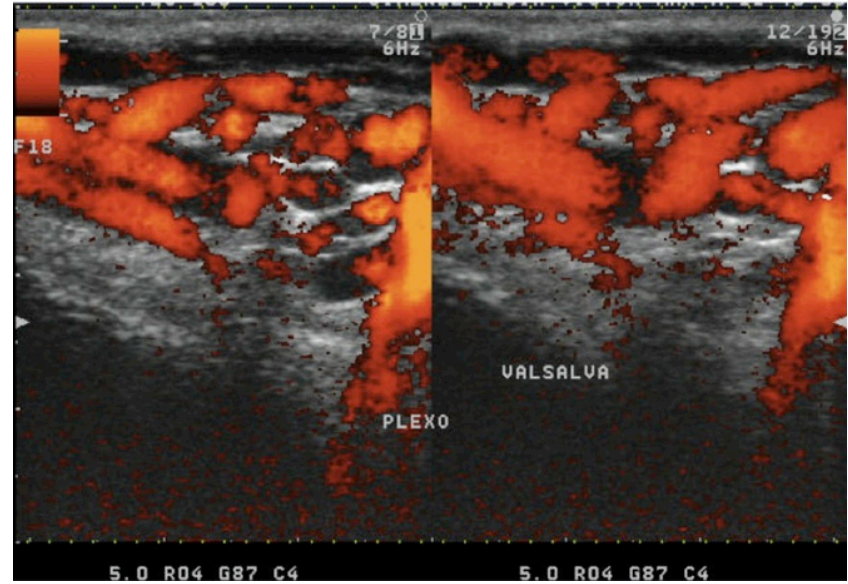

Figura 2 Imagen con modalidad Doppler color con maniobra de Valsalva, en el cual se confirma la ectasia del plexo pampiniforme.

derecha, inicialmente de las arterias aorta y mesentérica superior, ambas sin lesiones angiográficas, posteriormente por flebografía se identificó compresión extrínseca de la vena renal izquierda con vasos varicosos (fig. 3); se documentó un gradiente cavo-renal de $7 \mathrm{mmHg}$, se decidió realizar angioplastía periférica con implante de stent autoexpandible de nitinol en la vena renal izquierda, se colocó un introductor largo y curvo 9F, se avanzó el sistema de liberación Amplatzer ToqVue ${ }^{\circledR} 45^{\circ}$, ya una vez posicionada una guía Amplatzer super Stiff ${ }^{\oplus} 0.035 \times 260 \mathrm{~cm}$, se avanzó un stent autoexpandible Sentinol Biliar ${ }^{\circledR} 9 \times 42 \mathrm{~mm}$ con adecuado resultado angiográfico, asimismo sorprendió la reducción importante de la circulación colateral y finalmente, se evaluó el gradiente medio cavo-renal que se reduce a $0 \mathrm{mmHg}$, con lo que se concluyó el procedimiento como exitoso y sin complicaciones (fig. 4).

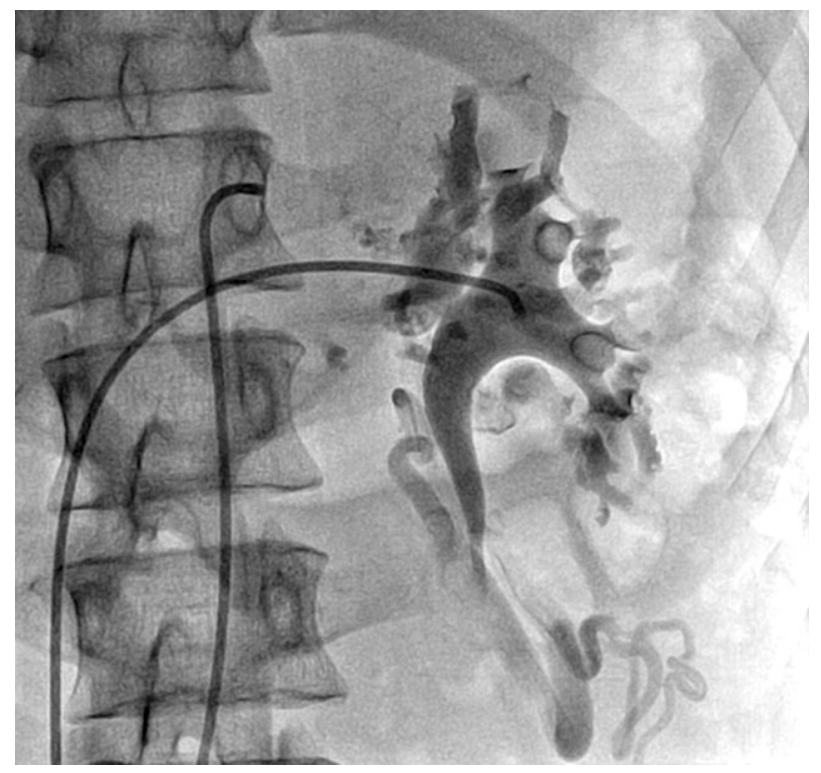

Figura 3 Flebografía selectiva de la vena renal izquierda en proyección posteroanterior, que muestra congestión venosa con importantes vasos colaterales y dilatados. 


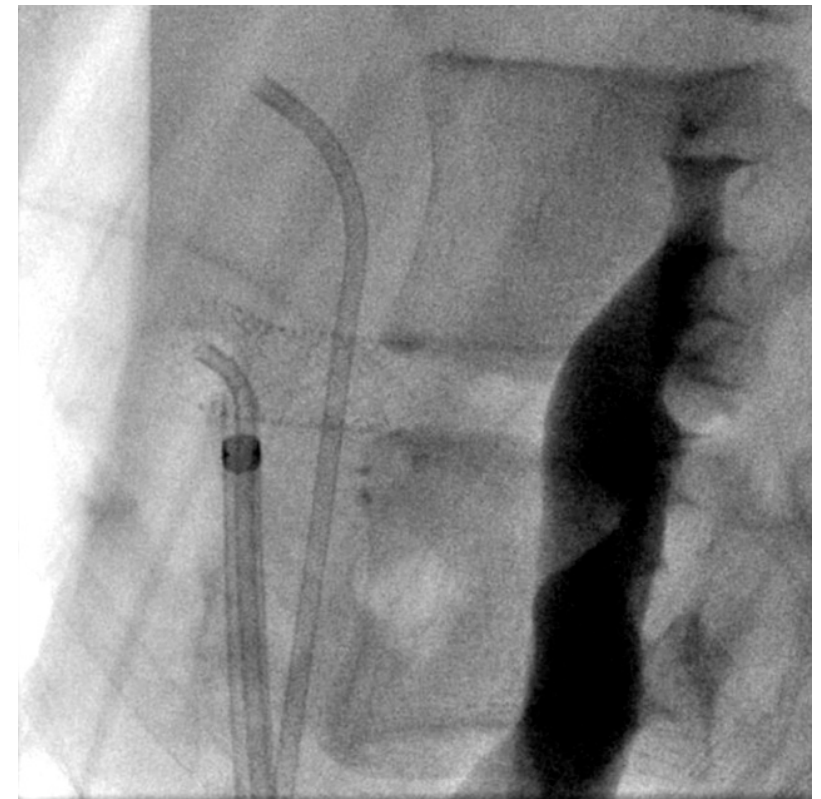

Figura 4 Proyección lateral, que muestra el resultado final del stent autoexpandible con adecuada apertura del mismo.

\section{Discusión}

El síndrome del cascanueces fue descrito por primera vez en 1950 por El Sadar y Mina, también Chait describió que la aorta y la arteria mesentérica superior forman los 2 brazos del cascanueces y que pueden potencialmente comprimir la vena renal izquierda; este fue denominado como síndrome del cascanueces por De Schepper ${ }^{7}$ en 1972. Se presenta por igual en ambos sexos, su prevalencia verdadera no se conoce; aparece en aquellos individuos en los que hay una compresión de la vena renal izquierda en la pinza que forma la arteria mesentérica superior y la aorta, de esta forma aquellos pacientes con ptosis renal, con escasa grasa perirrenal y lordosis lumbar acentuada presentan una predisposición anatómica, asimismo puede presentarse en el embarazo y puede empeorar durante el tercer trimestre. El síndrome de cascanueces invertido es la consecuencia sintomática de la entrada de la vena renal derecha entre la arteria mesentérica superior y la aorta ${ }^{11}$. También se ha descrito este fenómeno en el espacio retroaórtico por anomalía de trayecto de la vena renal izquierda, que queda atrapada entre la aorta y la columna lumbar ${ }^{6}$, esto da como consecuencia, hipertensión venosa retrógrada y la formación de circulación colateral compensadora ante la imposibilidad de un drenaje adecuado en la vena cava.

En nuestro caso, el dato clínico inicial fue la hematuria y leve proteinuria, lo cual fue estudiado y se descartó cada uno de los diagnósticos diferenciales como litiasis, malformaciones congénitas vasculares, tumores, infecciones, anomalías del parénquima o de la vía urinaria, entre otras. Se debe enfatizar que la primera herramienta diagnóstica es la semiología y la exploración física, si el paciente tiene síntomas de congestión pélvica y hematuria, la asociación de dolor en el flanco izquierdo con irradiación al área glútea, disconfort pélvico y várices pélvicas en una mujer, o varicocele en el varón, constituyen una fuerte base en el diagnóstico ${ }^{6,12,13}$. El apoyo de imagen con la TC y el US Doppler utilizados en este caso nos permitieron establecer el diagnóstico y pusieron de manifiesto las alteraciones vasculares venosas secundarias a la persistencia de la hipertensión venosa, si bien el diagnóstico puede sospecharse por Dop-

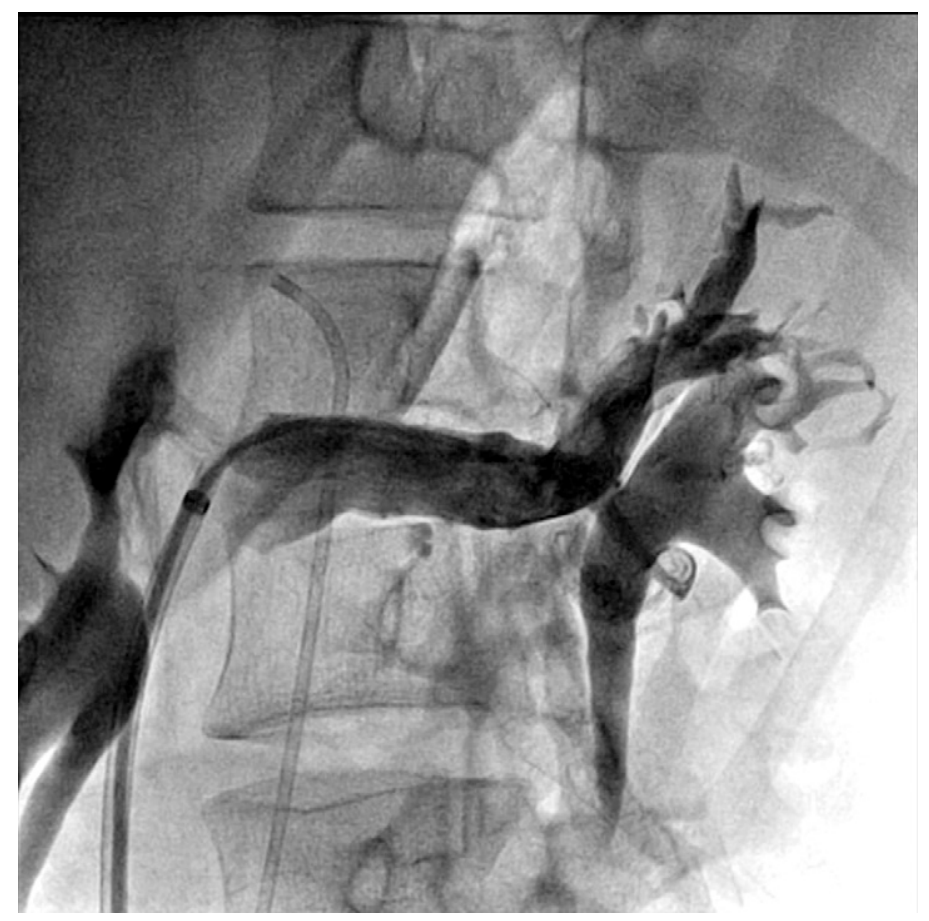

Figura 5 Flebografía selectiva de la vena renal izquierda con excelente resultado angiográfico y franca disminución de la circulación colateral y congestión venosa. 
pler venoso ${ }^{14}$, que nos permite determinar el diámetro y velocidad máxima de flujo en las porciones proximal y distal de la vena renal izquierda, la TC aporta información de la relación de la vena renal izquierda con la aorta y la arteria mesentérica superior y el estándar de oro diagnóstico se le considera a la venografía ${ }^{15,16}$. El tratamiento intervencionista con el implante de stent autoexpandible, no sólo tuvo el éxito angiográfico en el sitio de compresión extrínseca, con una clara reducción del gradiente venoso (reno-cava), sino también con inmediata reducción del calibre de las colaterales (fig. 5).

Aunque existen diferentes estudios como la serie publicada por Shanwen Chen, la literatura médica que existe acerca del tratamiento endovascular es alentadora y limitada para la evaluación definitiva del procedimiento, por lo cual deberá esperarse nuevas series para sustentar esta recomendación.

\section{Conclusión}

El síndrome de cascanueces es una causa infrecuente de hematuria microscópica cuyo tratamiento depende de la gravedad del cuadro clínico. El caso aquí presentado fue tratado exitosamente con un stent autoexpandible.

\section{Financiamiento}

No se recibió patrocinio de ningún tipo para llevar a cabo este artículo.

\section{Conflicto de intereses}

Los autores declaran no tener conflicto de intereses.

\section{Bibliografía}

1. Vidarte 0 , Salinas C, Quiroa F, et al. Hematuria macroscópica persistente causada por el "síndrome de cascanueces". Rev Med Hered. 2000;11:34-9.
2. Gorospe E, Aigbe M. Nutcracker Syndrome: a rare case of hematuria. Scientific World Journal. 2006;6:745-6.

3. Shokeir A, El Diatsi T, Ghoneim M. The nutcracker syndrome: new methods of diagnosis and treatment. $\mathrm{Br} \mathrm{J}$ Urol. 1994;74:139-43.

4. Vesga $F$, Acha $M$, Albisu A, et al. Vena renal retroaórtica. Presentación de un caso. Arch Esp Urol. 1994;47:285-7.

5. Santos A, Salgado R, Chiva V. Síndrome de cascanueces, a propósito de un caso y revisión de la literatura. Actas Urol Esp. 2003;34:123-8.

6. Scultetos A, Villavicencio A, Gillespie D. The nutcracker syndrome its role in the pelvic venous disorder. J Vasc Surg. 2001;34:812-9.

7. Youness E, Hassan J, Jaouad C, et al. Actitud expectante en el síndrome de cascanueces. Actas Urol Esp. 2009;33: 93-6.

8. Yong $\mathrm{B}$, Seong $\mathrm{H}$, Joo $\mathrm{H}$, et al. Nutcracker Syndrome: intravascular stenting approach. Nephrol Dial Transplant. 2000: 99-101.

9. Suwanabol P, Tefera G, Schwarze M. Syndromes associated with the deep veins: Phlegmasia Cerulea Dolens, May-Thurner Syndrome and Nutcracker Syndrome. Perspect Vasc Surg Endovasc Ther. 2010;22:223-30.

10. Chen S, Zhang $\mathrm{H}$, Shi $\mathrm{H}$, et al. Endovascular stenting for treatment of Nutcracker Syndrome: Report of 61 cases with long-term followup. J Urol. 2011;186:570-5.

11. Ekwenna O, Gorin MA, Castellan M, et al. Inverted Nutcracker Syndrome: a case of persistent hematuria and pain in the presence of a left-sided inferior vena cava. Scientific World Journal. 2011;11:1031-5.

12. Dellavedova T, Racca L, Ponzano RM, et al. Síndrome de cascanueces: comunicación de un caso. Rev Mex Urol. 2010;70: $51-4$.

13. Suárez R, Jiménez $P$, Castañeda $R$, et al. Megagastria $y$ megaduodeno asociado con Síndrome de arteria mesentérica superiory síndrome de cascanueces. Rev Esp Méd Quir. 2011;16: 51-5.

14. Yang W, Liu Y, Yang F. Meso-aortic compression of left renal vein revealed by multidetector computed tomography: nutcracker syndrome. Emerg Med J. 2007;24:636.

15. Natale F, Granato Ch, Arone L, et al. Nutcracker síndrome. Eur J Echocardiog. 2009;10:993.

16. Martínez J, García S, Herranz F, et al. Síndrome "nutcracker" o cascanueces: demostración mediante TAC helicoidal con reconstrucción "3D”' (VR). Actas Urol Esp. 2004;28:38-45. 